Descargo de responsabilidad: las opiniones expresadas en los manuscritos son responsabilidad exclusiva de los autores. No necesariamente reflejan las opiniones de la editorial ni la de sus miembros.

\title{
Trastorno por déficit de atención e hi- peractividad en la escuela primaria
}

\section{Attention deficit hyperactivity disorder in elementary school}

Recibido: $12 / 10 / 2021$

Publicado: 20/01/2022

Monica Miriam Guzmán Pérez miriamguzman50@gmail.com https://orcid.org/0000-0002-2129-4357

Filiación institucional de los autores Maestría en Neurociencia con énfasis en Neurocognición Universidad de San Carlos de Guatemala $\begin{array}{r}\text { Magdalena Chocoj González } \\ \text { arboledamcg@gmail.com }\end{array}$
https://orcid.org/0000-0002-0395-6185

$\begin{array}{r}\text { Magdalena Chocoj González } \\ \text { arboledamcg@gmail.com }\end{array}$
https://orcid.org/0000-0002-0395-6185

Corina Irene González de la Roca corinagonzalez.url@gmail.com https://orcid.org/0000-0003-3020-2773

Idania Marisol Alfaro Mendez ialfaromz@gmail.com https://orcid.org/0000-0001-8333-6631

Alejandro Rios Ramírez josealexrios@gmail.com https://orcid.org/0000-0002-5343-6436

\section{Referencia}

Guzmán Pérez, M. M., Chocoj González, M., González de la Roca, C. I., Alfaro Mendez , I. M., \& Rios Ramírez, A. (2022). Trastorno por déficit de atención e hiperactividad en la escuela primaria. Revista Académica Sociedad Del Conocimiento Cunzac, 2(1), 1-8. DOI: https://doi.org/10.46780/sociedadcunzac.v2i1.6

\section{Resumen}

OBJETIVO: contribuir con la formación de los docentes de las escuelas del municipio de Agua Blanca, Jutiapa sobre el trastorno por déficit de atención e hiperactividad (TDAH). MÉTODO: se realizó un estudio de tipo descriptivo cuantitativo con los docentes del municipio de Agua Blanca Jutiapa, siendo la muestra de 132 docentes. RESULTADOS: el estudio indica que los docentes no tienen capacitaciones sobre el tema de neurodesarrollo. Sobre trastorno por déficit de atención e hiperactividad, el 34.8\% de docentes han recibido una capacitación anual. Esto indica que los docentes no tienen el conocimiento específico para el abordaje de este trastorno en el aula. CONCLUSIÓN: Ios docentes tienen noción sobre TDAH, pero es necesario mayor formación, con el objetivo de discernir este trastorno del neurodesarrollo y referir a los alumnos con sospecha de TDAH con profesionales especializados. No cuentan con equipo multidisciplinario para la atención y seguimiento adecuado en casos de estudiantes con TDAH. Sin embargo, al tener solo conocimiento general de TDAH es difícil determinar si las estrategias de abordaje básicas para su apoyo son tomadas en cuenta en dichas adecuaciones. 


\section{Palabras clave}

docente, alumno, trastorno por déficit de atención con/ sin Hiperactividad (TDAH), neuroeducación, conocimientos

\section{Abstract}

OBJECTIVE: to contribute to the training of teachers in the schools of the municipality of Agua Blanca, Jutiapa on attention deficit and hyperactivity disorder (ADHD). METHOD: a quantitative descriptive study was carried out with teachers from the municipality of Agua Blanca Jutiapa, with a sample of 132 teachers. RESULTS: the study indicates that teachers do not have training on the subject of neurodevelopment. Regarding attention deficit hyperactivity disorder, $34.8 \%$ of teachers have received annual training. This indicates that teachers do not have the specific knowledge to address this disorder in the classroom. CONCLUSION: teachers have a notion about ADHD, but further training is necessary, in order to discern this neurodevelopmental disorder and refer students with suspected ADHD to specialized professionals. They do not have a multidisciplinary team for adequate care and follow-up in cases of students with ADHD. However, having only general knowledge of ADHD it is difficult to determine if the basic approach strategies for its support are taken into account in said adjustments.

\section{Keywords}

teacher, student, attention deficit disorder with / without hyperactivity, neuroeducation and knowledge

\section{Introducción}

La neurociencia permite a través de la neuroeducación, profundizar el conocimiento de las actividades educativas actuales en Guatemala. Esto ha generado la necesidad de conocer sobre el abordaje de calidad proporcionado a niños con TDAH en la etapa escolar. El objeto de realizar el presente estudio es concretar si el sistema educativo de Guatemala brinda atención oportuna a niños con esta condición.

El trastorno por déficit de atención con hiperactividad (TDAH), es un trastorno del neurodesarrollo, identificándose una serie de alteraciones neurocognitivas y conductuales, en la que se puede hacer mención la inhibición, la memoria de trabajo, la velocidad de procesamiento, la activación, el lapso de atención, la velocidad de procesamiento, la toma de decisiones y el retraso en la aversión (Rohde et al., 2019, p. 26).

Por lo que este estudio se orientó a contribuir con la formación de los docentes de las escuelas del municipio de Agua Blanca, sobre el trastorno por déficit de atención e hiperactividad (TDAH), debido a que los alumnos y docentes comparten varias horas en la escuela, el docente es quien, por su observación diaria e interacción se percata de las diferencias de algún alumno respecto al resto del grupo. (Domínguez, 2017). Por otra parte, se presenta un predominio del procesamiento global sobre el procesamiento detallado, es decir que los niños con TDAH evidencian mayores dificultades para evaluar y analizar el entorno y sus características (Valda et al., 2018, 124). 
Por ende, se buscó la caracterización del conocimiento de TDAH en los docentes, el identificar los factores institucionales que influyen por parte del docente en la comprensión y el abordaje del TDAH en el aula y la implementación de estrategias básicas para el abordaje de TDAH en el aula utilizadas por los docentes de primaria del municipio de Agua Blanca Jutiapa.

\section{Materiales y métodos}

Se diseñó un cuestionario de 16 preguntas cerradas, enfocadas en el conocimiento del trastorno por déficit de atención e hiperactividad como principal foco de estudio. Además de datos sobre la existencia de equipo multidisciplinario que atienda casos de necesidades educativas especiales y formación docente en el neurodesarrollo.

\section{Resultados}

Los datos recolectados fueron procesados, utilizando las herramientas estadísticas automatizadas a través de un Google Forms. El análisis de resultados se realizó con el total de la muestra encuestada.

Tabla 1. Capacitación del Docente/estrategias

\begin{tabular}{|c|c|c|}
\hline \multicolumn{3}{|c|}{ Capacitaciones relacionadas a neurodesarrollo } \\
\hline Ninguna & 80 & $60.9 \%$ \\
\hline 1 al año & 46 & $34.8 \%$ \\
\hline $1 \mathrm{al} \mathrm{mes}$ & 6 & $4.3 \%$ \\
\hline 1 en el semestre & 0 & 0 \\
\hline \multicolumn{3}{|c|}{ Capacitación sobre TDAH } \\
\hline $\begin{array}{l}\text { He recibido capacitaciones sobre } \\
\text { temas de Necesidades Educativas } \\
\text { Especiales de forma generalizada }\end{array}$ & 70 & $52.2 \%$ \\
\hline No & 57 & $43.5 \%$ \\
\hline $\mathrm{Si}$ & 5 & $4.3 \%$ \\
\hline \multicolumn{3}{|c|}{ Atención a estilos de aprendizaje } \\
\hline Si & 132 & $100 \%$ \\
\hline No & 0 & 0 \\
\hline \multicolumn{3}{|c|}{ Adecuaciones curriculares } \\
\hline $\mathrm{Si}$ & 80 & $60.9 \%$ \\
\hline Siempre lo hago & 35 & $26.1 \%$ \\
\hline Algunas veces (no me queda tiempo) & 17 & $13 \%$ \\
\hline No & 0 & 0 \\
\hline
\end{tabular}

Fuente: elaboración propia con base a la boleta de recolección de datos.

Los resultados denotan la deficiencia en la divulgación de temas relacionados con el neurodesarrollo y por supuesto en temas específicos como lo es el TDAH. La frecuencia de formación anual es del $34.8 \%$ que equivale a una población de 46 docentes que se capacitan en este 
tiempo, pero lamentablemente el $60.9 \%$ indica que no reciben capacitaciones sobre educación inclusiva y trastornos del neurodesarrollo, esto equivale a 80 personas de las 132 encuestadas.

De los 132 encuestados solo 5 docentes que corresponde al $4.3 \%$ de la muestra, ha recibido capacitaciones específicas sobre trastorno por déficit de atención e hiperactividad, en contraparte del $52.2 \%$ que equivale a 70 de los casos han recibido algún tipo de capacitación. Teniendo conocimientos generalizados sobre temas del neurodesarrollo y atención a necesidades educativas especiales. Lo cual evidencia la necesidad de mayor difusión acerca del conocimiento y manejo en el aula del trastorno por déficit de atención e hiperactividad. Los $132(100 \%)$ docentes encuestados reconocen que sus alumnos tienen diferentes formas de aprender y que no adquieren el conocimiento al mismo ritmo y en el tiempo. El $80 \%$ realizan adecuaciones curriculares o modificaciones en su método de enseñanza cuando identifican en sus alumnos algún problema o dificultad de aprendizaje o conductas.

Tabla 2. Factores institucionales que influyen en el conocimiento y abordaje del TDAH desde la perspectiva multidisciplinaria.

\begin{tabular}{|c|c|c|}
\hline $\begin{array}{l}\text { Características identificadas TDAH } \\
\text { por docentes }\end{array}$ & Cantidades & Porcentajes \\
\hline \multicolumn{3}{|l|}{ Inatención } \\
\hline Dificultad para prestar atención continua & 126 & $95.7 \%$ \\
\hline Errores frecuentes en sus tareas & 86 & $65.2 \%$ \\
\hline $\begin{array}{l}\text { Dificultad para comenzar y terminar } \\
\text { tareas }\end{array}$ & 63 & $47.8 \%$ \\
\hline Inatención en tareas & 46 & $34.8 \%$ \\
\hline $\begin{array}{l}\text { Evitar actividades de esfuerzo mental } \\
\text { sostenido }\end{array}$ & 46 & $34.8 \%$ \\
\hline \multicolumn{3}{|l|}{ Impulsividad } \\
\hline $\begin{array}{l}\text { No sabe esperar turno para intervenir en } \\
\text { una conversación o juego }\end{array}$ & 80 & $60.9 \%$ \\
\hline $\begin{array}{l}\text { Responden antes de que le termine de } \\
\text { preguntar }\end{array}$ & 75 & $56.5 \%$ \\
\hline Rompe las normas de clase & 57 & $43.5 \%$ \\
\hline $\begin{array}{l}\text { Tienen mala organización en su } \\
\text { escritorio }\end{array}$ & 57 & $43.5 \%$ \\
\hline \multicolumn{3}{|l|}{ Hiperactividad } \\
\hline $\begin{array}{l}\text { Se mantiene en constante movimiento } \\
\text { de piernas y/o manos }\end{array}$ & 92 & $69.6 \%$ \\
\hline $\begin{array}{l}\text { Interrumpe la concentración de sus } \\
\text { compañeros }\end{array}$ & 80 & $60.9 \%$ \\
\hline $\begin{array}{l}\text { Se levanta de su pupitre interrumpiendo } \\
\text { el trabajo de los demás. }\end{array}$ & 63 & $47.8 \%$ \\
\hline Se sale de clase y no hace caso & 28 & $21.7 \%$ \\
\hline Es muy travieso & 23 & $17.4 \%$ \\
\hline \multicolumn{3}{|l|}{ Aprendizaje } \\
\hline Lectoescritura & 86 & $65.2 \%$ \\
\hline Memoria & 57 & $43.5 \%$ \\
\hline Cálculo & 46 & $34.8 \%$ \\
\hline Comprensión lectora & 46 & $34.8 \%$ \\
\hline Alteraciones de la mecánica de la lectura & 17 & $13 \%$ \\
\hline
\end{tabular}

Fuente: elaboración propia con base a la boleta de recolección de datos. 
Al evaluar la característica de inatención, el $95.7 \%$ que corresponde a 126 de los docentes manifiesta que la dificultad para prestar atención continua es la caracterización que predomina; seguido por los errores frecuentes en sus tareas, un $65.2 \%$. que denota a 86 docentes. Así mismo el $47.8 \%$ que identifica a 63 de los encuestados respondieron; que la dificultad para comenzar y terminar tareas es un hallazgo recurrente entre los alumnos. Se evidenció que un $34.8 \%$ que representa a 46 docentes, que han observado inatención en tareas y actividades que requieren un mayor esfuerzo mental sostenido.

En referencia a la característica de impulsividad, el no saber esperar turno para intervenir en una conversación de juego es el hallazgo más frecuente el cual corresponde en un $60.9 \%$ que equivale a 80 docentes y en un $56.5 \%$ que representa a 75 docentes los cuales indicaron que los niños responden antes de terminar de escuchar una pregunta, y un $43.5 \%$ que equivale a 57 encuestados indicó que los niños rompen las normas de clase y tienen una mala organización en sus útiles.

De acuerdo con el análisis de la característica de hiperactividad, en un $69.6 \%$ que equivale a 92 de los docentes indican que los niños están en constante movimiento de las manos o de las piernas, un $60.9 \%$ correspondiente a 80 encuestados indicaron que este grupo de niños interrumpen en sus tareas a sus compañeros. Y el $47.8 \%$ representando a 63 docentes, indicó que los niños se levantan de su lugar interrumpiendo el trabajo de los demás.

En la característica del aprendizaje, la lectoescritura fue la habilidad más afectada manifestada por el $65.2 \%$ señalado por 86 docentes, seguido de las dificultades para memorizar con un $43.5 \%$ señalado por 57 . Las deficiencias en las áreas de comprensión lectora y cálculo fueron seleccionadas por un $34.8 \%$ que corresponde a 46 de los encuestados. La alteración de la mecánica de la lectura fue el área con menor afectación, reflejada por el $13 \%$ equivalente a17docentes.

Tabla 3. Factores institucionales que influyen en el conocimiento y abordaje del TDAH desde la perspectiva multidisciplinaria.

\begin{tabular}{lcc}
\hline Equipo multidisciplinario & Cantidades & Porcentajes \\
\hline Ninguno & 115 & $87 \%$ \\
\hline Maestros facilitadores (Maestro Sombra) & 11 & $8.7 \%$ \\
\hline Psicólogo educativo & 6 & $4.3 \%$ \\
\hline Orientador escolar & 0 & 0 \\
\hline Terapeuta de educación especial. & 0 & 0 \\
\hline Trabajador social & 0 & 0 \\
\hline
\end{tabular}

Fuente: elaboración propia con base a la boleta de recolección de datos. 
En referencia a los profesionales con los que cuenta el establecimiento donde laboran, se obtuvo como resultado que 115 docentes que equivale al $87 \%$ no cuentan con ningún tipo de acompañamiento, únicamente 11 de los entrevistados cuentan con algún tipo de apoyo profesional para el abordaje del TDAH; que equivale solo al $8.7 \%$ maestros sombra y $4.3 \%$ representado por 6 docentes indican tener apoyo de Psicólogo educativo. Es evidente la necesidad de poder reforzar el seguimiento de casos con niños con TDAH en la escuela del nivel primario, debido a que no se cuenta con personal calificado o equipo multidisciplinario para atender casos de TDAH en la mayoría de las escuelas, siendo un panorama de atención y seguimiento preocupante.

\section{Discusión}

Los profesores del municipio de Agua Blanca, Jutiapa del sector oficial; no cuentan con formación pertinente a la temática del TDAH. Sus conocimientos en atención a trastornos del neurodesarrollo son de forma generalizada. El conocimiento del trastorno, de sus características fundamentales, de sus criterios diagnósticos y sobre todo los trastornos asociados que pueden presentar ayudarían al profesorado a adaptar el trabajo con este alumnado para llevar a cabo una intervención adecuada y evitar futuros problemas como la baja autoestima y la frustración (Martínez, 2017, p. 256).

En relación con capacitaciones en el área de neurodesarrollo y en específico en TDAH, vemos que los docentes del municipio de Agua Blanca Jutiapa no cuentan con formación pertinente a la temática del TDAH. En el estudio de (López et al., 2018), sobre los conocimientos de los maestros de educación primaria sobre el trastorno por déficit de atención/hiperactividad, concluyeron que los maestros poseen un conocimiento parcial del TDAH. Identificamos que los conocimientos en atención a trastornos del neurodesarrollo son de forma generalizada. Vemos la necesidad de una mayor formación sobre este tema. Esta misma necesidad se observa en la investigación de Jarque, Tárraga y Miranda (2007) citado por Domínguez (2017), quienes realizaron un estudio sobre los profesionales de la educación y sus conocimientos sobre TDAH. Ellos ven la necesidad esencial de ampliar la formación específica sobre este trastorno en los cursos de formación continua a docentes y en los estudios de Magisterio. En el presente estudio los docentes marcan en cada una de las características del trastorno por déficit de atención e hiperactividad como mayor dificultad la falta de atención continua, estar en constante movimiento, el no saber esperar turno para intervenir en una conversación o juego y la dificultad en lectoescritura. Vemos que (Orellana, 2018) cita a George Still, pediatra inglés, quien en 1902 publicó un artículo donde describe el patrón conductual, destacando la falta de atención, búsqueda de gratificación, falta de control inhibitorio, poca preocupación por las consecuencias de sus acciones. Esta conducta no estaba vinculada a patrones de crianza. El $87 \%$ (115) de los docentes indican que las escuelas del municipio no cuentan con personal de apoyo necesario para el abordaje multidisciplinario de la atención y seguimiento adecuado en casos de estudiantes con TDAH. El mejor tratamiento es aquel que conjuga no solo a un grupo de especialistas, sino también integra en el equipo de trabajo a los padres, psicólogos y escuelas que favorecen el mejor rendimiento y crecimiento de un pequeño. Este tratamiento integral debe incluir al pediatra. 


\section{Referencias}

Domínguez Martín, R. (2017). Actualidad formativa andaluza en trastorno por déficit de atención e hiperactividad. [Tesis Doctoral Inédita]. In Universidad de Sevilla, Sevilla. idus.us.es. https://hdl.handle.net/11441/74715

López - López, A., López Lafuente, A., Eirís-Puñal, J., Mulas, F., \& Cardo, E. (2018). Estudio de los conocimientos de los maestros de educación primaria sobre el trastorno por déficit de atención/hiperactividad. REV NEUROL, 66(1), 121-S126.

DOI: https://doi.org/10.33588/rn.66S01.2018018

Martínez Blanco, N. (2017). Una aproximación al TDAH. Revista Internacional de Apoyo a la Inclusión, Logopedia, Sociedad y Multiculturalidad., 3(1), 248-257. 2387-0907. https://revistaselectronicas.ujaen.es/index.php/riai/article/view/4264

Orellana Ayala, C. E. (2018). Historia del T.D.A.H. https://carlosorellanaayala.com/2018/08/18/ historia-del-t-d-a-h/?fbclid=IwAR2jGv8ZdJ51TOrrJYfWFVIfMyJm1p_-K6zOLffwjT9q1wVPPhEFqdUdv7I

Rohde, L. A., Buitelaar, J. K., Gerlach, M., \& Faraone, S. V. (Eds.). (2019). La Federación Mundial de TDAH Guía. ARTMED EDITORA LTDA. 978-85-8271-578-9. http://cpo-media.net/ ADHD/2019/ebook\%20spanish/HTML/files/assets/common/downloads/publication.pdf

Valda Paz, V., Suñagua Aruquipa, R., \& Coaquira Heredia, R. K. (2018). Estrategias de intervención para niños y niñas con TDAH en edad escolar. Revista de Psicología, 20, 119 -179. http://scielo.org.bo/scielo.php?pid=s222330322018000200010\&script=sci_arttext

\section{Sobre los autores}

Grupo de investigadores de la Maestría en Neurociencias con énfasis en Neurocognición, Universidad de San Carlos de Guatemala.

\section{Financiamiento de la investigación}

Con recursos propios de los investigadores

\section{Declaración de intereses}

Declaran no tener ningún conflicto de intereses, que puedan haber influido en los resultados obtenidos o las interpretaciones propuestas.

\section{Declaración de consentimiento informado}

El estudio se realizó respetando el Código de ética y buenas prácticas editoriales de publicación. 


\section{Derechos de uso}

Copyright@ 2022 por Monica Miriam Guzmán Pérez, Magdalena Chocoj González, Corina Irene González de la Roca, Idania Marisol Alfaro Mendez, Alejandro Rios Ramírez

Este texto está protegido por una licencia Creative Commons 4.0

Este texto está protegido por la Licencia Creative Commons Atribución 4.0 Internacional.

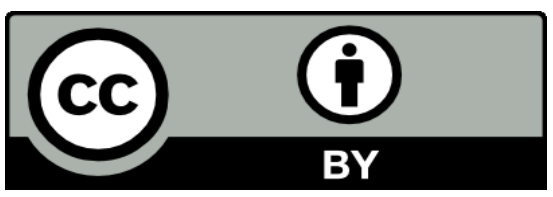

Este texto está protegido por una licencia

Creative Commons 4.0.

Es libre para compartir, copiar y redistribuir el material en cualquier medio o formato y adaptar el documento, remezclar, transformar y crear a partir del material para cualquier propósito, incluso comercialmente, siempre que cumpla la condición de atribución: debe reconocer el crédito de una obra de manera adecuada, proporcionar un enlace a la licencia, e indicar si se han realizado cambios. Puede hacerlo en cualquier forma razonable, pero no de forma tal que sugiera que tiene el apoyo del licenciante o lo recibe por el uso que hace. 